\section{SAT0013 IL-1A AND ADAMTS5 MEDIATED TISSUE DAMAGE IN HUMAN CARTILAGE EXPLANTS LEADS TO GENERATION OF TLR2 ACTIVATING DAMPS}

N. Sharma ${ }^{1,2}$, T.G. Christiansen ${ }^{3}$, M. Karsdal ${ }^{2}$, A.-C. Bay-Jensen ${ }^{2}$, C.S. Thudium ${ }^{2}$ ${ }^{1}$ Department of Biomedical science, University of Copenhagen, Copenhagen; ${ }^{2}$ Nordic Bioscience, Herlev, ${ }^{3}$ Orthopaedicsurgery unit, Gentofte University Hospital, Gentofte, Denmark

Background: The innate immune system is important for the initiation and development of joint diseases such as rheumatoid arthritis (RA) and Osteoarthritis (OA). Exogenous TLR (Toll like receptor) ligands are abundant in the synovial joints of RA and OA patients and induce the first immune response. Stimulation of TLRs results in an increased immune response and is characterised by induction of cytokines expression which in turn causes tissue damage and results in generation of more DAMPs. Hence, resulting in a self-perpetuating loop of TLR activation and TLR mediated tissue damage.

Objectives: The aim of this study was to investigate the effect of degradation fragments of human cartilage generated ex vivo or in vitro, on TLR-2 activation in a TLR-2 reporter cell system.

Methods: Human cartilage biopsies retrieved from OA patients undergoing total knee replacement at Gentofte hospital were used to generate human explants (HEX). HEX were cultured for 21 days either without $(\mathrm{w} / \mathrm{o})$ treatment or in the presence of IL-1 $\alpha(10 \mathrm{ng} / \mathrm{ml}, 5 \mathrm{ng} / \mathrm{ml}$ and $2.5 \mathrm{ng} / \mathrm{ml})$. Unconditioned media incubated without cartilage tissue was used as control. Tissue degradation was verified by measuring the release of aggrecan neo-epitope biomarkers AGNx1 and FFGV into the conditioned media (CM). Correspondingly, human cartilage was digested with ADAMTS5 for different time intervals ( 16 hour, 24 hour and 88 hour) at $37^{\circ} \mathrm{C}$. The digested cartilage was removed by centrifugation and the supernatant was stored. Crushed cartilage in buffer alone was used as a control set up for each time point and buffer alone was subtracted as background. The CM and digested media (DM) was tested in the SEAP (secreted embryonic alkaline phosphatase) reporter gene based HEK hTLR2 cell line. The HEK null 1 parent cell line was used as a control. Pam3CSK4 was used as a positive control for the hTLR2 cell line.

Results: IL-1 $\alpha$ induced aggrecan degradation was confirmed by increased exAGNx1 and exFFGV release in the conditioned media compared to $\mathrm{w} / \mathrm{o}$ $(p<0.01)$ (figure 1a). CM from IL-1 $\alpha$ treated explants induced a significant signal in the hTLR2 cells compared to w/o CM or to UCM with IL-1 $\alpha$ ( $p<0.0001$ ). (figure $1 b)$. Similarly, DM showed a significant induction in the hTLR2 cells after 88 hours compared to undigested human cartilage. $(\mathrm{p}<0.0001)$ (figure $1 \mathrm{c})$.

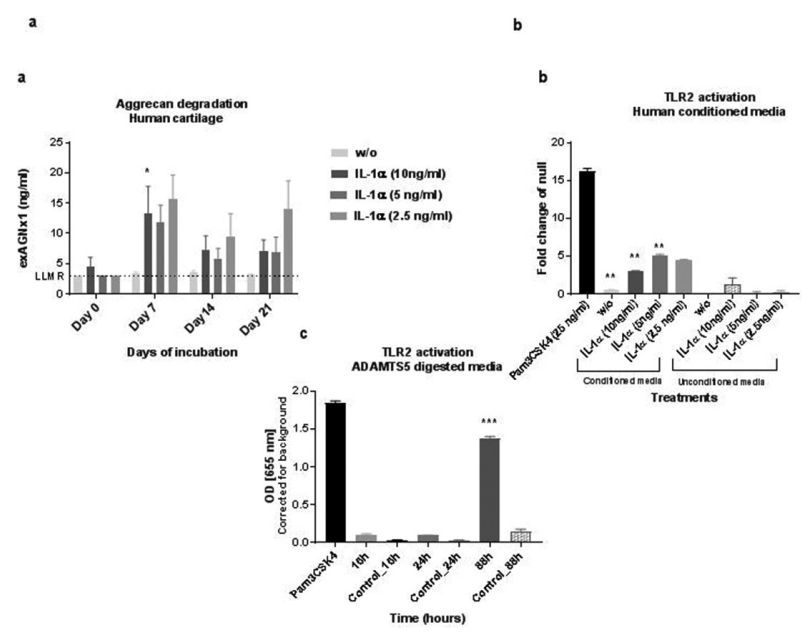

Conclusions: IL-1 $\alpha$ induced cartilage degradation leads to the release of aggrecan fragments into the $\mathrm{CM}$, and this $\mathrm{CM}$ as well as in vitro cleavage products from ADAMTS-5 digestion of human cartilage was able to activate the TLR2 receptor in vitro in a specialised reporter cell system. These data indicate that DAMPs may be released from human cartilage in the presence of pro-inflammatory cytokines and proteolytic enzymes. The released fragments can lead to TLR2 activation and cause further inflammation. These data suggest that DAMPs may play a role in the onset and maintenance of inflammation in diseases such as OA and RA.

Disclosure of Interest: None declared

DOI: 10.1136/annrheumdis-2018-eular.2715

\section{SAT0014 DRUG REPURPOSING TO BLOCK TLR4-ASSOCIATED INFLAMMATION IN OA CHONDROCYTES}

E. Franco-Trepat ${ }^{1}$ on behalf of Musculoskeletal Pathology Group, A. AlonsoPerez ${ }^{1}$ on behalf of Musculoskeletal Pathology Group, M. Guillan Fresco ${ }^{1}$ on behalf of Musculoskeletal Pathology Group, A. Jorge Mora ${ }^{1}$ on behalf of Musculoskeletal Pathology Group, V. López ${ }^{2}$ on behalf of NEIRID LAB, O. Gualillo ${ }^{2}$ on behalf of NEIRID LAB, R. Gómez on behalf of Musculoskeletal Pathology Group on behalf of Musculoskeletal Pathology Group. ${ }^{1}$ Musculoskeletal Pathology Group; ${ }^{2}$ Health Research Institute of Santiago de Compostela (IDIS), Santiago de Compostela, Spain

Background: The rising prevalence of rheumatic diseases in our society has caused a dramatic impact on the welfare of the population as well as becoming an economic burden to the health system. Osteoarthritis $(\mathrm{OA})$, the most common rheumatic disease, is defined by joint-space narrowing due to progressive cartilage degradation. Despite the growing knowledge in OA pathophysiology, no treatment has yet proved to be efficient enough. The activation of innate immune receptors, such Toll-like receptor 4 (TLR4), by damage-associated molecular patterns (DAMPS) has been involved in chondrocyte-mediated inflammatory responses. There are currently no available drugs aimed to block TLR4-mediated inflammatory responses. Nonetheless, there are already known drugs being employed in other indications that could have this activity; namely amitriptyline, naloxone, and thalidomide.

Objectives: Determine the ability of amitriptyline, naloxone and thalidomide to block TLR4-mediated innate immune responses in chondrocytes.

Methods: The effect of amitriptyline, naloxone and thalidomide on TLR4-mediated inflammatory responses was determined in mouse chondrogenic cell line (ATDC5) and in human primary OA chondrocytes. The mRNA expression of key inflammatory factors lipocalin-2 (LCN2), interleukin-6 (IL-6), and monocyte chemoattractant protein-1 (MCP-1) was studied by RT-PCR. Cell viability was tested using the methyl-thiazolyl-tetrazolium (MTT) reagent and nitrite accumulation (nitric oxide production) in cell culture media was assessed by Griess reaction and validated by determining nitric oxide synthase 2 (NOS-2) mRNA expression. Results: The co-stimulation of human OA chondrocytes with the TLR4 agonist LPS [100 $\mathrm{ng} / \mathrm{ml}]$ and amitriptyline $[1 \mu \mathrm{M}]$, reduced the mRNA expression of LCN2 (90\%), IL-6 (95\%) and MCP-1 (87\%). The pre-stimulation of naloxone [100 $\mu \mathrm{M}]$ with LPS also reduced the mRNA expression of LCN2 (53\%), IL-6 (78\%) and MCP-1 (79\%). Similar results LCN2 (63\%), IL-6 (74\%), and MCP-1 (78\%) were obtained upon the pre-stimulation of these cells with thalidomide [500 $\mu \mathrm{M}$ ]. The anti-inflammatory effect of these drugs on these pro-inflammatory factors was also observed but lowered in ATDC5 cells. Consistent with these results in ATDC5 cells these drugs also reduced the expression of mRNA NOS-2 gene expression as well as nitrite accumulation in the cell culture medium. At the studied concentrations, amitriptyline, naloxone and thalidomide did not affect to chondrocytes viability.

Conclusions: The data presented here have shown that amitriptyline, naloxone and thalidomide block TLR4 innate immune responses in human OA chondrocytes. These drugs have passed many toxicology and safety tests for their clinical use and could be ready for its repurposing in the management of TLR4-mediated OA cartilage inflammation.

Acknowledgements: This research is supported by Fondo de Investigación Sanitaria funded by the Instituto de Salud Carlos III and FEDER (PI16/01870 CP15/00007). R.G. is funded by the Instituto de Salud Carlos III through a Miguel Servet programme. R.G. is a member of the RETICS Programme, RD12/0009/ 0008 Instituto de Salud Carlos III (ISCIII).

Disclosure of Interest: None declared

DOI: 10.1136/annrheumdis-2018-eular.5369

\section{SAT0015 IDENTIFICATION AND CHARACTERISATIONOF HIGH MOLECULAR WEIGHT HMGB1 PROTEIN COMPLEXES: IMPLICATIONS FOR STRESS RESPONSE, INNATE IMMUNITY AND AUTOIMMUNE DISEASE}

W. Willis, L. Wang, T.T. Wada, M. Garder, O. Abdouni, G. Valiente, S. Agarwal, M. A. Freitas, L.-C. Wu, W.N. Jarjour. The Ohio State University, Columbus, USA

Background: High mobility group box one protein (HMGB1) is a chromatin associated protein, which in response to stress or injury translocates from the nucleus to the extracellular milieu to serve as an alarmin. HMGB1 has a remarkable ability to form complexes with proinflammatory molecules. This is underscored by the emerging role of HMGB1 in rheumatic diseases such as systemic lupus erythematosus (SLE), where the presence of HMGB1 in complex with endogenous nuclear 